\title{
Ensuring the Week Goes Smoothly - Improving Daily Surveillance Visualization
}

\author{
Roger Morbey ${ }^{\star 1}$, Alex J. Elliot ${ }^{1}$, Elizabeth Buckingham-Jeffery² and Gillian E. Smith ${ }^{1}$
}

${ }^{1}$ Public Health England, Birmingham, United Kingdom; ${ }^{2}$ Warwick University, Coventry, United Kingdom

\section{Objective}

To develop smoothing techniques for daily syndromic surveillance data that allow for the easier identification of trends and unusual activity independent of day of the week and holiday effects.

\section{Introduction}

Real-time syndromic surveillance requires daily surveillance of a range of health data sources. Most real-time data sources from health care systems exhibit large day of the week fluctuations as service provision and patient behaviour varies by day of the week. Regular day of the week effects are further complicated by the occurrence of public holidays (usually 8 per year in England), which can limit the availability of certain services and affect patient behaviour. Simple seven day moving averages fail to provide a smoothed trend around public holidays and can lead to false alarms or potentially delays in detection of outbreaks.

\section{Methods}

Data were used from four national syndromic surveillance systems (a non-emergency medical number, emergency department records, and information from family doctor in hours and unscheduled care consultations) coordinated by Public Health England. Day of the week effects were modelled in the absence of public holidays by calculating the percentage of a week's activity that occurs on each day of the week for a range of different syndromic indicators and syndromic surveillance systems. Simple statistical t-tests were used to check for the significance of differences between days of the week.

Syndromic data were examined to test how public holidays impacted on day of the week effects. Days immediately preceding and following holidays were also examined to identify any significant changes. Differences between public holidays based on the time of year and the number of holidays within a single year were also examined.

Smoothing techniques for different syndromic systems were developed to remove artificial spikes around public holidays in simple seven day moving averages.

\section{Results}

The impact of day of the week effects were found across all syndromic surveillance systems. Unsurprisingly GP in hours data reported very little activity at weekends and on holidays, whilst GP unscheduled care and telephone help line data activity was roughly double at weekends and holidays. Day of the week effects were much less clear in emergency department attendances but significant differences were still demonstrable.

Public holiday activity was similar to weekend activity in most cases, although activity on 25th December, Christmas day was considerably less than other holidays. Evidence was seen both of increased activity immediately prior to public holidays and on the first working day after a public holiday.

Seven day moving averages that are adjusted for bank holidays were shown to be effective in smoothing out artificial spikes in data.

\section{Conclusions}

Improved understanding of day of the week and public holiday effects enables improved modelling of baselines used in statistical detection algorithms, for instance 25th December should not be treated as having the same impact as other public holidays. Future work will also consider condition-specific differences where the case mix varies by day of the week and during holidays.

These improved smoothing techniques have enabled improved data visualization tools, enabling investigators to easily identify unusual activity during daily surveillance.

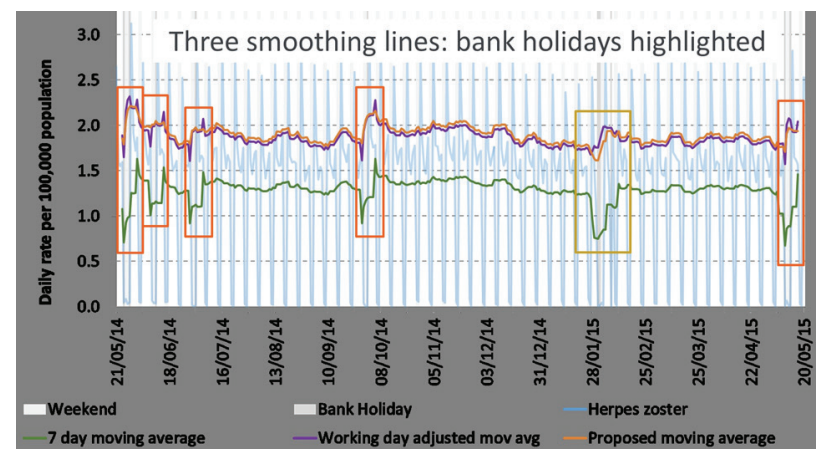

Example of improved smoothing for daily rates of Herpes Zoster consultations.

\section{Keywords}

Surveillance; visualisation; smoothing

\section{Acknowledgments}

We acknowledge support from: Royal College of Emergency Medicine, EDs participating in the emergency department system (EDSSS), Ascribe Ltd and L2S2 Ltd; OOH providers submitting data to the GPOOHSS and Advanced Heath \& Care; TPP and participating SystmOne practices and University of Nottingham, ClinRisk, EMIS and EMIS practices submitting data to the QSurveillance database; and NHS 111 and HSCIC for assistance and support in providing the anonymised call data that underpin the Remote Health Advice Syndromic Surveillance System. We thank the PHE Real-time Syndromic Surveillance Team for technical expertise. The authors received support from the National Institute for Health Research Health Protection Research Unit in Emergency Preparedness and Response. The views expressed are those of the authors and not necessarily those of the NHS, the NIHR, the Department of Health or Public Health England.

\section{${ }^{*}$ Roger Morbey}

E-mail: roger.morbey@phe.gov.uk 